\title{
Study of Aerobic Bacteriological Profile of Surgical Site Infections and their Antibiogram at Tertiary Care Center
}

\author{
Hema Panchalamarri and A. Renuka devi* \\ Department of Microbiology in Kurnool Medical College, Kurnool, India \\ *Corresponding author
}

\section{A B S T R A C T}

\begin{tabular}{|l|}
\hline Ke y w or d s \\
$\begin{array}{l}\text { nosocomial } \\
\text { infection, surgical } \\
\text { site infection, } \\
\text { antibiogram. }\end{array}$ \\
\hline Article Info \\
\hline $\begin{array}{l}\text { Accepted: } \\
\text { 24 August } 2019 \\
\text { Available Online: } \\
\text { 10 September } 2019\end{array}$ \\
\hline
\end{tabular}

Surgical site infection (SSI) is defined as an infection that develops within 30 days of surgery at the incision site. They are the second most common nosocomial infections causing significant morbidity, prolonged hospitalization, and death. To study the bacteriological profile of surgical site infections and their antibiogram. This is a study carried out in patients who underwent surgery from January 2018 to December 2018 in Obstetrics and Gynecology, General Surgery and Orthopaedic departments in Government general hospital, Kurnool. Culture, identification, and Antibiotic Susceptibility testing were performed using standard techniques. A total of 126 surgical wound samples were included in the study. 70 (55.5\%) were culture positive. The infection was found to be higher in females of $20-60$ years age group than in males. The most commonly isolated pathogens were Klebsiella (35.7\%) followed by Staphylococcus aureus (25.7\%) and Escherichia coli(25.7\%). Among gram-positive isolates, four strains (22.2\%) were Methicillinresistant, and eight strains (18.6\%) were ESBL producers among gram-negative isolates. There is a need for optimal perioperative and infection control practices to reduce the incidence of SSI's.

\section{Introduction}

Surgical site infection (SSI) is an infection that develops within 30 days after a surgical procedure or 1 year if an implant is placed and the infection appears to be related to surgery (1).These are one of the most common healthcare-associated infections (HAIs), causing substantial morbidity, with 2-11-fold higher mortality(2). Based on the depth of infection penetration into the wounds, SSI's are divided into three types - superficial incisional, deep incisional, and organ/space(3).

Most of the patients do not develop an infection at the surgical site due to the presence of strong host innate immunity, which eliminates the microbial contaminant at the surgical site. However, when host defense mechanisms fail to remove the microbial contamination compounded by large 
inoculum, the virulence of pathogens, all together pave the path to the development of SSI. Infectious agents causing SSI's may be acquired from exogenous sources or endogenous sources. Exogenous sources are those external to the patient, such as patient care personnel, visitors, patient care equipment, medical devices, or the health-care environment and endogenous sources are the patient's flora present on skin, nose, mouth, gastrointestinal tract, or vagina.

The occurrence of SSI following surgery depends on the type of wound (clean, cleancontaminated, contaminated or dirty), immune status of the host, presence or absence of a foreign body/prosthesis, and core body temperature fluctuations. They have been associated with complications such as increased readmission rates, increased hospital length of stay, enhanced overall costs, and increased morbidity and mortality rates(3)(4).

Surveillance data suggest that the types of causative organisms associated with SSI have not significantly changed over the past 10-15 years; however, the proportion of different types of causative organisms has changed. Antimicrobial-resistant organisms are causing an increasing proportion of SSIs, and there has been a rise in the number of infections caused by atypical bacterial and fungal organisms.

These changing proportions have been attributed to the increasing acuity of surgical patients, the increase in the number of immunocompromised patients, and the increasing use of broad-spectrum antibiotics(5).

This study aims to determine the bacteriological profile of SSIs and their antimicrobial susceptibility profile at our tertiary care center, which would help to institute better prophylactic measures and appropriate, timely and accurate therapeutic measures to reduce the cost of treatment and morbidity of the disease.

\section{Materials and Methods}

This was a prospective study conducted from January 2018 to December2018 in the department of Microbiology in Kurnool medical college, Kurnool.

One hundred and twenty-six samples were collected from patients in Obstetrics and Gynecology, General Surgery and Orthopaedic departments who had developed post-operative wound infections with purulent discharge and clinically diagnosed as postoperative sepsis.

Purulent materials were collected on sterile commercial cotton swabs aseptically and gently to avoid contamination of the specimens with normal microbial flora of the skin. Specimens were collected before redressing and administration of antibiotic therapy. Specimens were labeled, kept in a thermoflask containing ice and transferred immediately to the laboratory for bacteriological examination.

A smear was prepared and stained by Gramstaining for early presumptive diagnosis. The samples were inoculated onto Blood agar, Macconkey agar by streak method using Nichrome loop.The plates were incubated overnight at $37^{\circ} \mathrm{C}$ for $18-24$ hours. Identification of colonies was done by cultural characteristics and biochemical tests. Antimicrobial susceptibility tests were done on Mueller- Hinton agar by Kirby Bauerdisk diffusion method according to Clinical Laboratory Standards Institution (CLSI) guidelines(6).

The antibiotics used in the study are depicted in table-1. Phenotypic methods were used to detect and report methicillin-resistant Staphylococcus aureus and extended-spectrum beta-lactamases (ESBL)production in Gramnegative bacteria as per the CLSI guidelines(6). 


\section{Results and Discussion}

Out of one hundred and twenty-six samples processed, seventy samples (55.5\%) were culture positive. a Maximum number of patients $(80.1 \%)$ were from 20-60 years of age group. Age group-wise distribution is depicted in table-2. There was no sex predilection.

Among the 70 isolated aerobic bacteria, 52 (74.2\%) were gram-negative organisms and 18 (25.7\%) were gram-positive organisms. Gramnegative isolates included, Klebsiella pneumoniae $(\mathrm{n}=25,35.7 \%)$, Escherichia coli $(\mathrm{n}=18,25.7 \%), \quad$ Pseudomonas aeruginosa $(\mathrm{n}=8,11.4 \%)$, Proteus mirabilis $(\mathrm{n}=01,1.42 \%)$. Gram-positive organism included Staphylococcus aureus (25.7\%). Organisms isolated are depicted in table 3.

Antimicrobial susceptibility patterns of isolates belonging to genus Staphylococcus (n $=18)$, Enterobacteriaceae family $(\mathrm{n}=44)$, nonfermenter Gram-negative Bacilli $(\mathrm{n}=8)$, are depicted in Figures 1-3, respectively.

In the present study, rate of postoperative wound infection was $11.73 \%$, that is similar to the other studies conducted in India(7). In corroboration with earlier studies, we also found that age group, gender, and type of surgery are not significant factors in the development of SSI.

The most common cause of SSI per our study was Klebsiella (35.7\%), followed by E. coli (25.7\%)) and Staphylococcus aureus (25.7\%), Pseudomonas aeruginosa (11.4\%), Proteus (1.42\%). Elgohari et al., in his Surveillance ofSurgical Infections in NHS hospitals in England 2013-2014, also reported S. aureus $(16 \%)$ as the most frequent species causing SSI(8).He also reported an increasing trend of Enterobacteriaceae which accounted for $21 \%$ SSI in his study. Of the samples collected from SSI in the present study, $44.4 \%$ were culture negative, which suggests other causes of SSI such as anaerobic organisms, Mycoplasma hominis, or Ureaplasma urealyticum which are not looked for routinely. Reddy(9),in his work from Hyderabad, also reported that up to $30 \%$ SSI could be labeled as culture negative if the organisms as mentioned earlier are not looked for specifically. A limitation of the present study is that anaerobic organisms were not investigated which may have been a cause of culture-negative SSI.

The increasing reports of multidrug-resistant organisms being isolated from SSI are a cause for concern. More than $70 \%$ of the isolates belonging to Enterobacteriaceae family [Figure 1] were resistant to third-generation cephalosporins like ceftriaxone, ceftazidime and fluoroquinolones.18.6\% were phenotypically ESBL producers.

High degree $(>40 \%)$ of resistance was also seen against gentamicin, piperacillintazobactam and amoxiclav, thus precluding the use of all these drugs as prophylactic and therapeutic agents. The spread of these organisms or their resistance genes in and outside hospital environment is a cause of concern. Almost all isolates were susceptible to polymyxins. Various Indian and Southeast Asian workers have reported similar findings(10)(11)(12)(13).

Nonfermenting Gram-negative Bacilli are ubiquitous and are notoriously multidrugresistant. In the present study [Figure 3], more than $50 \%$ of isolates were resistant to piperacillin-tazobactam, ceftazidime and more than $30 \%$ were resistant to cefepime, ciprofloxacin, and gentamicin. Only $75 \%$ were susceptible to meropenem, and all the isolates were susceptible to colistin. An increase in the number of drug-resistant isolates is a global problem. 
Table.1 Antibiotics with disc content

\begin{tabular}{|l|c|l|c|}
\hline \multicolumn{2}{|l|}{ Antibiotics For Gram Positive Bacteria } & Antibiotics For Gram Negative Bacteria \\
\hline Antibiotic & Disc Content $(\mu \mathrm{g})$ & Antibiotic & Disc Content $(\mu \mathrm{g})$ \\
\hline Amoxiclav & $20 / 10$ & Amoxiclav & $20 / 10$ \\
\hline Erytromycin & 15 & Gentamicin & 10 \\
\hline Clindamycin & 2 & Ciprofloxacin & $100 / 10$ \\
\hline Gentamicin & 10 & Piperacillin+ tazobactum & 30 \\
\hline Vancomycin & 30 & Ceftazidime & $30 / 10$ \\
\hline Linezolid & 10 & Ceftazidime+ Clavulanic acid & 30 \\
\hline Cefoxitin & 30 & Ceftriaxone & 10 \\
\hline Doxycycline & 30 & Imipenem & 10 \\
\hline Teicoplanin & 30 & Colistin & \\
\hline
\end{tabular}

Table.2 Age -group-wise distribution of SSI

\begin{tabular}{|c|c|}
\hline Age ( in years) & No. of SSI (in \%) \\
\hline$<20$ & 7.14 \\
\hline $20-60$ & 80.1 \\
\hline$>60$ & 12.6 \\
\hline
\end{tabular}

Table.3 Distribution of various organisms isolated

\begin{tabular}{|c|c|}
\hline Organism & NUMBER OF SSI (\%) \\
\hline Klebsiella pneumoniae & 35.7 \\
\hline Escherechia coli & 25.7 \\
\hline Staphylococcus aureus & 25.7 \\
\hline Pseudomonas aeruginosa & 11.4 \\
\hline Proteus mirabilis & 1.42 \\
\hline
\end{tabular}

Fig.1 Antimicrobial susceptibility of Staphylococcus aureus

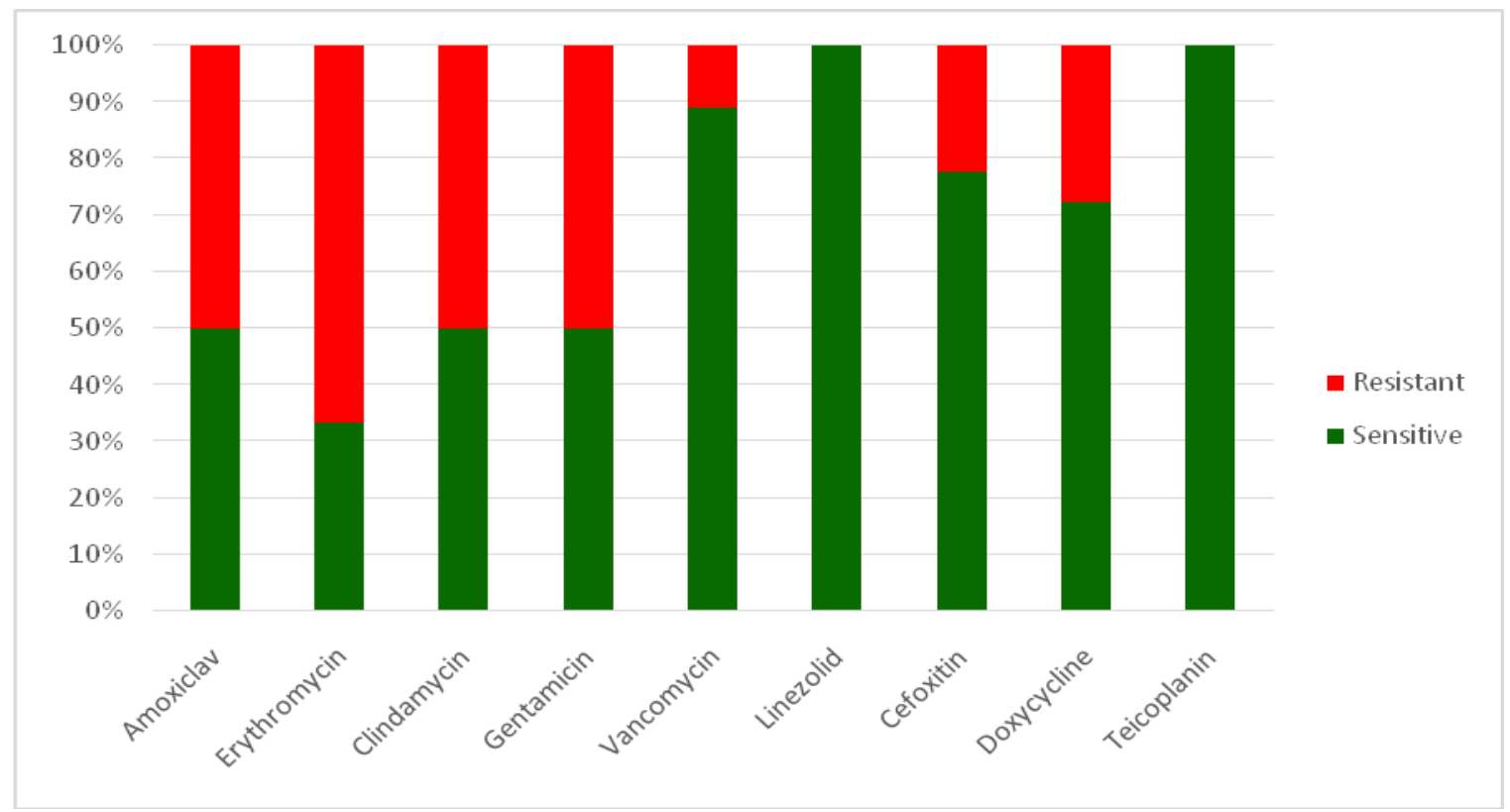


Fig.2 Antimicrobial susceptibility of isolates belonging to Enterobacteriaceae family



Fig.3 Antimicrobial susceptibility of non fermenters



Injudicious use of antimicrobial agents may lead to such a pattern, and we recommend combination therapy when such pathogens are isolated along with meticulous wound care and barrier nursing precautions to avoid the spread of these bugs(14). Although Indian 
workers have reported similar findings(13)(12),Europeanworkers reported a better susceptibility of $>90 \%$ toward carbapenems(15).

In the present study [Figure 2], 50\% isolates of Staphylococcus were resistant to penicillin, and notably, 22\% were cefoxitin (and therefore, methicillin) resistant. About 66\% were resistant to erythromycin with $50 \%$ being resistant to clindamycinincluding inducible resistance. All isolates were susceptible to linezolid and vancomycin with $100 \%$ susceptibility to teicoplanin(13).

The drug susceptibility findings of the present study show that antibiotics such as fluoroquinolones and third-generation cephalosporins would have minimal usefulness the prophylaxis or treatment of infections caused by Enterobacteriaceae since most of the patients receive third-generation cephalosporins as prophylactic agents. Non fermenters are reported to be highly resistant, and due to their ability to survive easily and form bio films, infections caused by them are challenging to treat(16)(17). It is known that peri operative prophylaxis can decrease the incidence of wound infection(18).Isolation of such multidrug-resistant strains is a cause of concern due to a limited repertoire of available antimicrobial agents.

There is an alarming increase in infections caused by antibiotic-resistant bacteria. Lack of uniform antibiotic policy and indiscriminate use of antibiotics may have lead to emergence of resistant bacterial strains. Based on our findings, we recommend a few protocols to be followed to prevent and reduce SSI, first hand hygiene and thorough infection control protocol to reduce the spread of microbes and plasmid-mediated drug resistance, the second - antibiotic stewardship and tailor-made prophylactic policies based on local susceptibility data, the third - maintenance of the infrastructure of operation theater and wards, and finally - a close collaboration between microbiologists and surgeons(19)(20).

\section{References}

Protocol for surgical site infection surveillance with a focus on settings with limited resources [Internet]. 2018 [cited 2019 Jul 16]. Available from: http://apps.who.int/bookorders.

Klevens RM, Edwards JR et.al. Estimating healthcare-associated infections and deaths in U.S. Hospitals in 2002. Public Health, 122:160-166. R 2007.

Centers for Disease Control and Prevention."National Healthcare Safety Network(NHSN) Patient Safety Component Manual. CDC A (2019).

Akhter MSJ, Verma R, Madhukar KP, Vaishampayan AR, Unadkat PC. Incidence of surgical site infection in postoperative patients at a tertiary care center in India. $\mathbf{J}$ Wound Care [Internet]. 2016 Apr 2 [cited 2019 Jul 16];25(4):210-7. Available from: http://www.ncbi.nlm.nih.gov/pubmed/ 27064370

Sievert DM, Ricks P, Edwards JR, Schneider A, Patel J, Srinivasan A, et al., Antimicrobial-resistant pathogens associated with healthcare-associated infections: summary of data reported to the national healthcare safety network at the centers for disease c 2009-2010. Infect Control Hosp Epidemiol 2013;34:1-4., 8.

26th TC\& LSI (CLSI). CSMPS for AST, ed. Wayne PTC and LSI 2016., 10.

Suchitra JB LNS site infections. Risk factors and antimicrobial sensitivity patterns. AJMR 2009;3:185-9. A from http://www. academicjournals. org/ajmr. [Last cited on $2010 \mathrm{~N} \mathrm{03].}$

Elgohari S, Mihalkova M, Wloch C, Saei A, 
Harris R, Charlett A, et al., Surveillance of surgical site infections in NHS hospitals in England 2013/14. London: Public Health England; 2014.

RBM of culture-negative surgical site infections. J of M and AS 2012;2:2-6. A from: http:// jmas. in/sites/default/files/articles/Manageme nt\%20of\%20Culture\%2.

Sohn AH, Parvez FM, Vu T, Hai HH, Bich NN, Le Thu TA, et al., Prevalence of surgical-site infections and patterns of antimicrobial use in a large tertiarycare hospital in Ho Chi Minh city VICHE 2002;23:382-7.

Anvikar A, Deshmukh A, Karyakarte R, Damle A, Patwardhan N, Malik A et al., O year prospective study of 3280 surgical wounds. IJMM 1999;17:12932. A from http://www. ijmm. org/article. $\quad$ asp?issn=025.0857; year $=1999$; volume $=17$; issue $=3$; $\mathrm{s}$ page $=129$; page $=132 ;$ aulast $=$ Anvikar; $t$ уре $=0$.

Kakati B, Kumar A, Gupta P, Sachan P TBS site abdominal wound infections: $\mathrm{E}$ at a NI tertiary care hospital. IA of CM 2013;14:13-9. A from: http://medind. nic. in/jac/t13/i1/jact13i1p13. pdf. [Last accessed on 2018 D 14].

Negi V, Pal S, Juyal D, Sharma MK, Sharma N. Bacteriological profile of surgical site infections and their antibiogram: A study from resource-constrained rural setting of Uttarakhand state IJCDR 2015;9:DC17-20.

Hemant Singhal KKW infection. OHT ancient $\mathrm{E}$ was the first civilization to have trained clinicians to treat physical ailments. eMed GS 2009. SI (Larchmt) 2009; 10:323-31.

Giacometti A, Cirioni O, Schimizzi AM, Del Prete MS, Barchiesi F, D'Errico MM et al., E and microbiology of surgical wound infections. JCM 2000;38:91822.

Song F GAA prophylaxis in colorectal surgery: A systematic review of randomized controlled, Surg trials. BJ, 1998;85:1232-41.

Olson MM, Lee JT Jr. Continuous, 10-year wound infection surveillance. Results, advantages and unanswered questions. AS 1990;125:794-803.

Ashby E, Haddad FS, O'Donnell E WAH will surgical site infection be measured to ensure "high-quality care for all"? JBJSB 2010;92:1294-9.

Gorecki P, Schein M, Rucinski JC WLA administration in patients undergoing common surgical procedures in a community teaching hospital: $\mathrm{T}$ chaos continues. WJS 1999;23:429-32.

Sawyer RG PTW infections. SCNA 1994;74:519-36.

\section{How to cite this article:}

Hema Panchalamarri and Renuka devi, A. 2019. Study of Aerobic Bacteriological Profile of Surgical Site Infections and their Antibiogram at Tertiary Care Center. Int.J.Curr.Microbiol.App.Sci. 8(09): 2667-2673. doi: https://doi.org/10.20546/ijcmas.2019.809.308 